Palavras chave:

Colorimetria

Eucalyptus grandis

Sistema CIE L*a*b*

Termorretificação

Histórico:

Recebido 23/ I / 20 I I

Aceito 25/1 I/2013

Keywords:

Colorimetry

Eucalyptus grandis

CIE L*a*b* system

Heat treatment

Correspondência: rosileigar@ufrrj.br
Rosilei Aparecida Garcia', Juliana de Oliveira Lopes', Wanessa Aparecida Santos'

\section{MODIFICAÇÃO DA COR ORIGINAL DA MADEIRA DE Eucalyptus grandis ATRAVÉS DE TRATAMENTOS TERMORRETIFICADORES}

RESUMO: Neste estudo, objetivou-se determinar a modificação da cor original da madeira de Eucalyptus grandis Hill ex. Maiden após a termorretificação. Amostras de madeira foram termorretificadas sob diferentes condições de temperatura (I80, 200, 215 e $230^{\circ} \mathrm{C}$ ) e tempo ( 15 minutos, 2 e 4 horas). As análises colorimétricas foram realizadas por meio do sistema CIE L*a*b* com o auxílio do espectrofotômetro Color Eye XTH$X$-Rite 200d. Todos os tratamentos termorretificadores causaram uma alteração na cor original da madeira. As madeiras termorretificadas apresentaram menores valores de $L^{*}$ (luminosidade), quando comparadas à madeira não tratada (controle), caracterizando o escurecimento da madeira, principalmente para as condições mais severas de temperatura e tempo. As coordenadas cromáticas a* e b* apresentaram comportamentos diferentes, dependendo da combinação temperatura-tempo. A modificação da cor original da madeira possibilitou a criação de novos padrões de cor, podendo agregar maior valor à madeira estudada.

\section{MODIFICATION OF THE ORIGINAL COLOR OF THE Eucalyptus grandis WOOD BY HEAT TREATMENTS}

ABSTRACT: The objective of this study was to determine the modification of original color of Eucalyptus grandis Hill ex. Maiden wood after heat-treatment. Wood samples were heat-treated under different temperatures $\left(180,200,215\right.$ and $\left.230^{\circ} \mathrm{C}\right)$ and time conditions ( 15 minutes, 2 and 4 hours). Color analysis were performed on the CIE L*a*b* system by using a Color Eye XTH-X-Rite 200d spectrophotometer. All heat treatments promoted an alteration of the original color of wood. Heat-treated woods presented lower L* (lightness) values than untreated wood (control), characterizing the wood darkness, mainly for more severe conditions of temperature and time. Chromatic coordinates ( $\mathrm{a}^{*}$ and $\mathrm{b}^{*}$ ) showed different behaviors depending on the temperature-time combination. The modification of the original color of the wood allowed the creation of new color patterns, which can add greater value to the studied wood.
DOI:
Universidade Federal Rural do Rio de Janeiro - Seropédica, Rio de Janeiro, Brasil 


\section{INTRODUÇÃO}

A termorretificação é um tratamento aplicado à madeira e seus derivados, visando à obtenção de produtos de maior estabilidade dimensional e maior durabilidade natural. Além dessas características, o tratamento também agrega à madeira menor teor de umidade de equilíbrio, alteração no teor de extrativos, maior isolamento térmico, menor resistência mecânica, menor resistência ao desgaste e à abrasão, menor rigidez, menor resistência à dureza, odor persistente e alteração da cor original (GARCIA et al., 20I2; KAMDEM et al., 2002; VIITANIEMI; JÄMSÄ, 1994).

Entre essas características, a alteração da cor original pode agregar maior valor ao produto final. Em países da Europa e América do Norte, o tratamento tem sido aplicado em madeiras de cores claras e de menor valor comercial tais como Pinus spp e Picea spp visando à obtenção de produtos de maior valor agregado. De acordo com Bekhta e Niemz (2003), Mitsui et al. (200I) e Mitsui (2004, 2006), a madeira termorretificada apresenta uma vantagem estética, já que esta adquire uma cor similar às madeiras tropicais, sendo, portanto, apreciada para inúmeras aplicações. O tratamento ocasiona o escurecimento da madeira, o qual tende a aumentar em função da temperatura e do tempo de tratamento (AKYILDIZ et al., 2009; AYADI et al., 2003; MILITZ, 2002; SUNDQVIST; MORÉN, 2002; SUNDQVIST, 2004).

A alteração da cor da madeira após a termorretificação pode ser explicada pela combinação de vários fatores, sendo eles: (a) a dissolvição, oxidação e decomposição dos extrativos e outros componentes químicos; (b) a formação de produtos oxidativos, tais como quinonas e produtos da degradação das hemiceluloses e lignina, os quais migram às superfícies da madeira; (c) a remoção ou migração dos extrativos e compostos nutritivos tais como açúcares de baixo peso molecular e aminoácidos em direção às superfícies da madeira; e (c) o aumento relativo no teor de lignina após o tratamento, em decorrência, principalmente da degradação das hemiceluloses (AKYILDIZ et al., 2009; BEKHTA; NIEMZ, 2003; ESTEVES et al., 2008; KAMKE, 2006; SUNDQVIST; MORÉN, 2002; THEANDER et al., 1993; TJEERDSMA et al., 1998; VARGA; VAN DER ZEE, 2008). Segundo Varga e Van der Zee (2008), as madeiras com grandes quantidades de extrativos solúveis em água apresentam alterações mais intensas de cor após o tratamento.

Alguns autores sugerem que a cor da madeira termorretificada pode ser ainda utilizada como um indicador das modificações ocorridas nas suas propriedades físicas e mecânicas (BEKHTA; NIEMZ, 2003; BOURGOIS et al., 199I; PATZELT et al., 2003). Bekhta e Niemz (2003) observaram uma correlação significativa entre a variação total da cor e a resistência da madeira de Picea sp. termorretificada sob diferentes condições de temperatura e tempo. Patzelt et al. (2003) também encontram uma boa correlação entre a perda de massa e as variáveis de cor L* (luminosidade) e $\mathrm{h}^{*}$ (ângulo de tinta) da madeira termorretificada. Schnabel et al. (2007) encontraram correlações importantes entre as variáveis de cor e a resistência à flexão para madeiras termorretificadas de Fraxinus excelsior e Fagus sylvatica e afirmam que a colorimetria pode ser utilizada como uma ferramenta no controle de qualidade, durante o processo de termorretificação.

Conduziu-se este estudo, com o objetivo de determinar a alteração da cor original da madeira de Eucalyptus grandis Hill ex. Maiden após a termorretificação, visando a contribuir para uma melhor compreensão das modificações ocasionadas na madeira sob diferentes condições de temperatura e tempo.

\section{MATERIAL E MÉTODOS}

\section{Material e tratamento de termorretificação}

Seis árvores de Eucalyptus grandis Hill ex. Maiden, com 23 anos de idade, foram coletadas de um plantio com espaçamento de $3 \times 2 \mathrm{~m}$, pertencente à empresa Quimvale Florestal Ltda, localizada na Região Sul do Rio de Janeiro. À partir das toras foram obtidas tábuas com dimensões de $200 \times 12 \times 5 \mathrm{~cm}$ (comprimento $\times$ largura $x$ espessura), as quais foram processadas na serraria LPZ Artefatos de Madeira Ltda e secas ao ar livre no Laboratório de Secagem de Madeira do Departamento de Produtos Florestais da Universidade Federal Rural do Rio de Janeiro-UFRRJ, Seropédica-RJ.

As tábuas foram redimensionadas e amostras medindo $400 \times 120 \times 50 \mathrm{~mm}$ (comprimento $\times$ largura $x$ espessura) foram submetidas ao tratamento de termorretificação sob diferentes condições de temperatura e tempo. A termorretificação foi realizada em uma estufa ELETROlab ${ }^{\circledR}$, modelo 403, com capacidade de $0,1 \mathrm{~m}^{3}$, dimensões internas e externas de $90 \times 98 \times 55 \mathrm{~cm} \mathrm{e} 50 \times 50 \times 40 \mathrm{~cm}$, respectivamente. Foram utilizadas 4 temperaturas $\left(180,200,215\right.$ e $\left.230^{\circ} \mathrm{C}\right)$ e 3 períodos de tempo ( 15 minutos, 2 e 4 horas), resultando em 12 tratamentos termorretificadores. As amostras foram introduzidas na estufa a uma temperatura inicial de $60^{\circ} \mathrm{C}$ e o tempo de aquecimento até a temperatura 
final não foi considerado. $O$ teor de umidade inicial das amostras foi de 12 à 14\%. Após os tratamentos, as amostras foram acondicionadas em câmara climática à $20^{\circ} \mathrm{C}$ e $65 \%$ de umidade relativa até atingir o teor de umidade de equilíbrio. As amostras termorretificadas foram comparadas com amostras controles.

\section{Análises colorimétricas}

As análises colorimétricas das madeiras termorretificadas e controles foram realizadas, a partir de amostras de $5 \times 5 \times 5 \mathrm{~cm}$, no espaço de cor L*a*b*, conhecido como sistema CIE L*a*b*, definido pela norma CIE-Commission Internationale de l'Éclairage (1976). As coordenadas de cor L*, a* e b* foram determinadas por meio do espectrofotômetro Color Eye XTH-X-Rite 200d, com iluminante D65 e ângulo de $10^{\circ}$, em temperatura ambiente de $22^{\circ} \mathrm{C}$ e $60 \%$ de umidade relativa, acoplado a um microcomputador disponível no Laboratório de Tecnologia da Madeira da Universidade de Brasília-UNB (Brasília, DF). A coordenada $L^{*}$ representa a luminosidade, a qual varia de zero (preto) a 100 (branco), enquanto que a* e b* representam as coordenadas cromáticas e variam de -60 à +60. No espaço tridimensional CIE L*a*b*, os sinais positivos e negativos significam: $+a^{*}$ aumento na cor vermelha, $-\mathrm{a}^{*}$ aumento na cor verde, $+\mathrm{b}^{*}$ aumento na amarela e -b* aumento na cor azul (KONICA MINOLTA SENSING, 1998).

Os valores de saturação da cor ( $\left.C^{*}\right)$ e ângulo de tinta (h) foram calculados para caracterizar a cor da madeira termorretificada pelas equações I e 2 .

$$
\begin{aligned}
& \mathrm{C}^{*}=\sqrt{\left(\mathrm{a}^{*}\right)^{2}+\left(\mathrm{b}^{*}\right)^{2}} \\
& \mathrm{~h}=\tan ^{-1}\left(\frac{\mathrm{b}^{*}}{\mathrm{a}^{*}}\right)
\end{aligned}
$$

A variação total da cor da madeira após os tratamentos termorretificadores também foi determinada por meio da equação 3 , sendo $\Delta \mathrm{E}$ : variação total da cor da madeira após a termorretificação; $\Delta \mathrm{L}^{*}, \Delta \mathrm{a}^{*}$ e $\Delta \mathrm{b}^{*}$ : variação das variáveis $L^{*}, a^{*}$ e $b^{*}$, respectivamente.

$$
\Delta \mathrm{E}=\sqrt{\left(\Delta \mathrm{L}^{*}\right)^{2}+\left(\Delta \mathrm{a}^{*}\right)^{2}+\left(\Delta \mathrm{b}^{*}\right)^{2}}
$$

A variação de cada coordenada foi determinada pela diferença entre o valor médio da coordenada da madeira original (controle) e o valor médio da coordenada da madeira termorretificada, tal como realizado por outros autores (DUBEY et al., 20II; GONZÁLEZ-
PENA; HALE, 2009; OYARCE, 2006; SUNDQVIST; MORÉN, 2002).

Foram efetuadas 20 observações para cada tratamento (I2 tratamentos termorretificadores + controle) nas seções longitudinais radial e tangencial de cada amostra, para cada variável de cor ( $L^{*}, a^{*}$ e b*).

\section{Análises estatísticas}

Os dados obtidos foram submetidos a uma análise de variância (ANOVA), com o auxílio do programa SAS $^{\circledR}$ (Statistical Analysis System). Quando o valor F foi significativo $(\alpha=0,05)$, aplicou-se o teste de média de Duncan $(\alpha=0,05)$, a fim de determinar as diferenças significativas entre os tratamentos termorretificadores.

\section{RESULTADOS E DISCUSSÃO}

Os resultados obtidos para as coordenadas colorimétricas no espaço L*a*b* da madeira termorretificada e controle de Eucalyptus grandis são apresentados na Tabela I. Para todos os tratamentos termorretificadores, a coordenada $L^{*}$ (luminosidade) apresentou valores significativamente menores que 0 controle, tanto na seção radial quanto na tangencial, caracterizando o escurecimento da madeira após a termorretificação (Tabela I). Nas temperaturas de 200,215 e $230^{\circ} \mathrm{C}$ há um escurecimento cada vez mais acentuado com o aumento do tempo de tratamento, exceto para a temperatura de $180^{\circ} \mathrm{C}$ em que a luminosidade foi menor para o tratamento de 2 horas. Os tratamentos realizados sob condições mais severas de temperatura e tempo, principalmente à 215 e $230^{\circ} \mathrm{C}$ durante 2 e 4 horas apresentaram os menores valores de L*. Esses resultados corroboram com estudos realizados por outros autores (AHAJJl et al., 2009; AKYILDIZ et al., 2009). Segundo Varga e Van Der Zee (2008), dentre as causas da alteração da cor da madeira durante o tratamento térmico, podemos citar a dissolução, oxidação e decomposição dos extrativos e outros componentes químicos. Segundo Chen et al. (20।2), a descoloração da madeira durante o tratamento térmico é decorrente das reações de condensação e oxidação, principalmente.

No caso de madeiras com grandes quantidades de extrativos solúveis em água, as alterações de cor são ainda mais intensas após o tratamento. Os compostos extrativos presentes nas madeiras de folhosas e responsáveis pela mudança da cor das mesmas são: os terpenos, fenóis, taninos, entre outros. (ISHIGURI et al. 2003). 
TABELA I Valores médios das variáveis colorimétricas da madeira de Eucalyptus grandis termorretificadas e controle para as seções radial e tangencial.

TABLE I Means values of the color variables of the control and heat-treated Eucalyptus grandis wood on radial and tangential sections.

\begin{tabular}{|c|c|c|c|c|c|c|c|c|c|c|c|c|}
\hline \multirow{2}{*}{ Tratamento } & \multicolumn{5}{|c|}{ Radial } & \multicolumn{5}{|c|}{ Tangencial } & \multirow{2}{*}{$\begin{array}{c}\Delta \mathrm{E} \\
\text { radial }\end{array}$} & \multirow{2}{*}{$\begin{array}{c}\Delta \mathrm{E} \\
\operatorname{tang}\end{array}$} \\
\hline & $L^{*}$ & $\mathrm{a}^{*}$ & $b^{*}$ & C* & $\mathrm{h}$ & $L^{*}$ & $\mathrm{a}^{*}$ & $b^{*}$ & C* & $\mathrm{h}$ & & \\
\hline Eontrole & & & & & & & & & & & - & - \\
\hline $180^{\circ} \mathrm{C} / 15 \mathrm{~min}$ & & & & & & & & & & & 8,10 & 8,72 \\
\hline $180^{\circ} \mathrm{C} / 2 \mathrm{~h}$ & & & & & & & & & & & 16,44 & 10,24 \\
\hline & & & & & & & & & & & 8,83 & 5,30 \\
\hline $200^{\circ} \mathrm{C} / 15 \mathrm{~min}$ & & & & & & & & & & & 3,93 & 4,09 \\
\hline $200^{\circ} \mathrm{C} / 2 \mathrm{~h}$ & $\begin{array}{l}62,15^{\mathrm{e}} \\
(2,90)\end{array}$ & & & & & & & & & & 12,84 & 4,10 \\
\hline $200^{\circ} \mathrm{C} / 4 \mathrm{~h}$ & & & & & & & & & & & 16,93 & $|4,7|$ \\
\hline $215^{\circ} \mathrm{C} / 15 \mathrm{~min}$ & $\begin{array}{l}70,92^{\mathrm{bc}} \\
(0,92)\end{array}$ & & & $\begin{array}{l}20,68^{d} \\
(0,55)\end{array}$ & $\begin{array}{l}59,10^{\mathrm{b}} \\
(2,46)\end{array}$ & & & & & & 3,95 & 4,72 \\
\hline $215^{\circ} \mathrm{C} / 2 \mathrm{~h}$ & $\begin{array}{l}46,83^{8} \\
(5,39)\end{array}$ & $\begin{array}{l}12,80^{c} \\
(0,35)\end{array}$ & & & & & & & & & 28,09 & 20,12 \\
\hline $215^{\circ} \mathrm{C} / 4 \mathrm{~h}$ & $\begin{array}{l}47,00 \mathrm{~g} \\
(2,31) \\
\end{array}$ & $\begin{array}{l}13,78^{\mathrm{b}} \\
(0,54)\end{array}$ & $\begin{array}{l}20,59^{\circ} \\
(1,57)\end{array}$ & $\begin{array}{l}20,93^{\mathrm{d}} \\
(4,93)\end{array}$ & & & & & & & 27,99 & 28,74 \\
\hline $230^{\circ} \mathrm{C} / 15 \mathrm{~min}$ & $\begin{array}{l}69,22^{c} \\
(2,44)\end{array}$ & $\begin{array}{l}10,88^{f} \\
(1,19)\end{array}$ & $\begin{array}{l}17,72^{\text {ed }} \\
(0,64)\end{array}$ & $\begin{array}{l}20,27^{d} \\
(1,95)\end{array}$ & $\begin{array}{l}58,46^{b c} \\
(2,61)\end{array}$ & & & & $\begin{array}{l}20,27^{d} \\
(1,95)\end{array}$ & $\begin{array}{l}57,67^{f g} \\
(1,56)\end{array}$ & 5,68 & 3,37 \\
\hline $230^{\circ} \mathrm{C} / 2 \mathrm{~h}$ & $\begin{array}{l}41,20^{h} \\
(2,36)\end{array}$ & $\begin{array}{l}11,74^{d} \\
(0,54)\end{array}$ & $\begin{array}{l}18,31^{\mathrm{d}} \\
(1,77)\end{array}$ & $\begin{array}{l}22,\left.9\right|^{\mathrm{bc}} \\
(0,96)\end{array}$ & $\begin{array}{l}57,23^{\text {bcd }} \\
(1,45)\end{array}$ & $\begin{array}{l}52,17^{\mathrm{e}} \\
(4,06)\end{array}$ & $\begin{array}{l}12,29^{c} \\
(0,38)\end{array}$ & $\begin{array}{l}19,33^{b} \\
(1,02)\end{array}$ & $\begin{array}{l}20,\left.9\right|^{b c} \\
(0,96)\end{array}$ & $\begin{array}{l}57,\left.5\right|^{\mathrm{bc}} \\
(1,27)\end{array}$ & 33,62 & 20,08 \\
\hline $230^{\circ} \mathrm{C} / 4 \mathrm{~h}$ & $\begin{array}{l}30,98 \\
(1,83)\end{array}$ & $\begin{array}{l}8,48^{8} \\
(0,82)\end{array}$ & $\begin{array}{l}12,10^{f} \\
(1,36)\end{array}$ & $\begin{array}{l}13,78^{e} \\
(2,23)\end{array}$ & & & & & & $\begin{array}{l}58,66^{a} \\
(0,88)\end{array}$ & 44,39 & 29,60 \\
\hline Valor F & 194,86** & $30,63 * *$ & 28,01 *** & $35,25 * *$ & 3,78 *** & $35,64 * *$ & $14,67 * *$ & 14,46 ** & 17,80 *** & $5,05 * *$ & --- & --- \\
\hline
\end{tabular}

L*: luminosidade. $\mathrm{a}^{*}$ e $\mathrm{b}^{*}$ : variáveis cromáticas. $\mathrm{C}^{*}$ : saturação da cor. h: ângulo de tinta. $\Delta \mathrm{E}$ : variação total da cor após a termorretificação. Valores entre parêntesis = desvio padrão. ** Significativo a $99 \%$ de probabilidade. Dentro de uma mesma coluna, tanto na seção radial como na tangencial, comparando controle e tratamentos, médias com a mesma letra não são estatisticamente diferentes entre si segundo o teste de Duncan a $95 \%$ de probabilidade.

Observou-se, também, maiores valores de L* (madeira mais clara) na seção radial que na seção tangencial (Figura IA). Gonçalez et al. (2006) e Ribeiro (2009) observaram a mesma variação de cor entre as faces radial e tangencial para madeiras de Eucalyptus grandis e Eucalyptus cloeziana.

A coordenada a* apresentou valores positivos, variando entre 8,48 e 14,45 e entre 7,94 e 13,84 para as seções radial e tangencial, respectivamente. Para a coordenada $b^{*}$, foram encontrados valores entre 12,10 e $21,7 \mid$ e entre $\mid I, 2 I$ e $2 I, 45$ para as seções radial e tangencial, respectivamente (Tabela I). As madeiras termorretificadas à $230^{\circ} \mathrm{C}$ por 4 horas, apresentaram os menores valores de $\mathrm{a}^{*} \mathrm{e} \mathrm{b}^{*}$ para ambas as seções. Esses resultados estão de acordo com aqueles encontrados por Schnabel et al. (2007) e Moura e Brito (20l I), os quais observaram valores menores de $a^{*}$ e $b^{*}$ para as madeiras tratadas a altas temperaturas. Segundo Moura e Brito (20II) supõem que a redução na cor vermelha está associada, provavelmente, à volatilização de alguns compostos fenólicos que conferem a cor vermelha do eucalipto quando a mesma é aquecida.

Para os tratamentos efetuados a condições menos severas de temperatura e tempo, as variáveis cromáticas (a* e b*) apresentaram um comportamento diferente, dependendo da combinação temperaturatempo, apresentando, ora valores significativamente maiores ora menores, quando comparados à madeira 

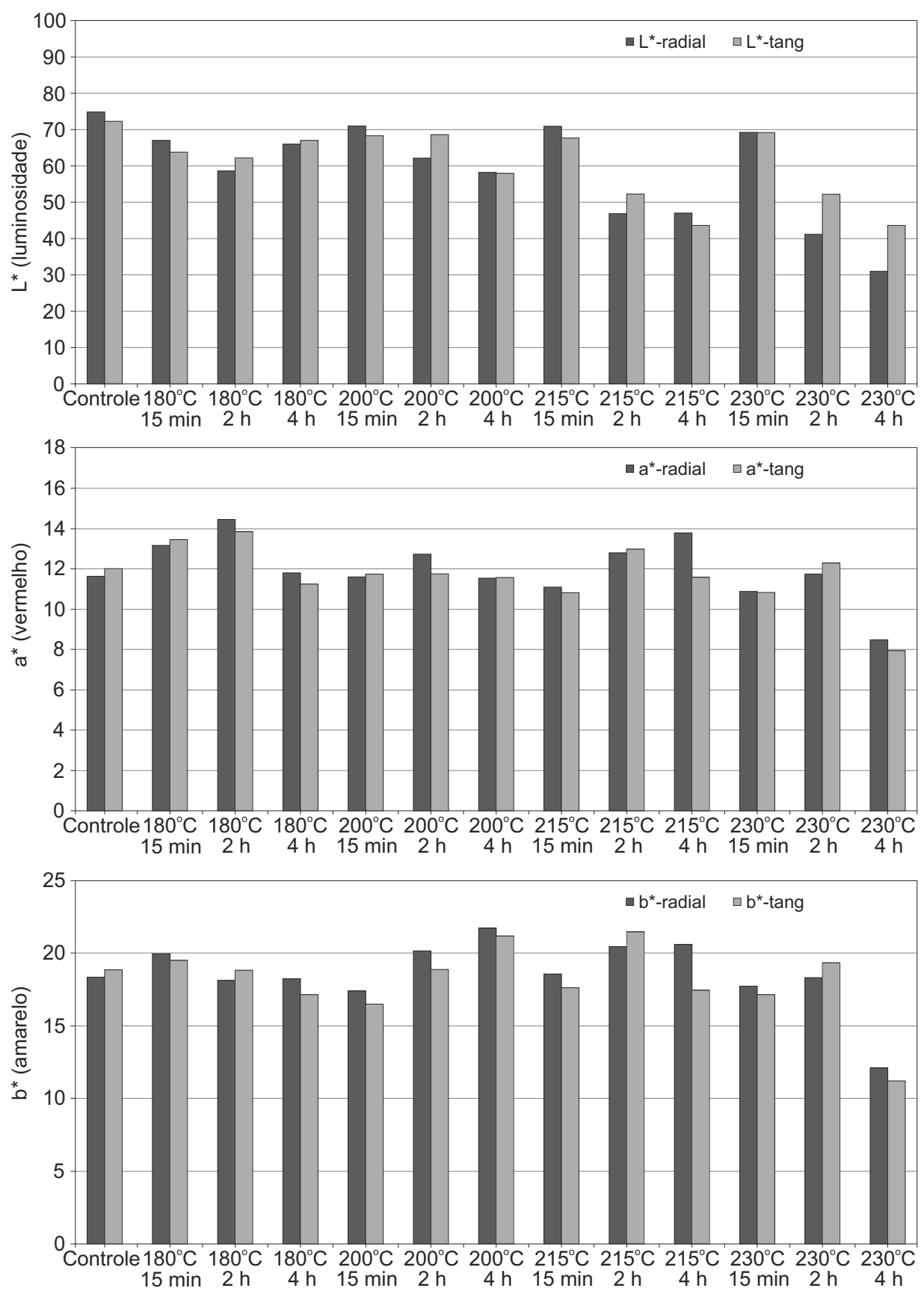

FIGURA I Valores médios das variáveis colorimétricas L*, a* e b* obtidos para as madeiras termorretificadas e controle de Eucalyptus grandis nas seções radial e tangencial.

FIGURE I Means values of the color variables L*, a* and b* of heat-treated and control Eucalyptus grandis wood on radial and tangential sections.

controle (Tabela I). Resultados similares foram observados por Schnabel et al. (2007) e Bourgois et al. (1991). Schnabel et al. (2007) obtiveram um aumento no valor de $\mathrm{a}^{*}$ para madeiras de Fraxinus excelsior e Fagus sylvatica tratadas a temperaturas de baixa e média intensidade, enquanto que, o valor de $\mathrm{a}^{*}$ permaneceu inalterado para tratamentos mais severos; o valor de

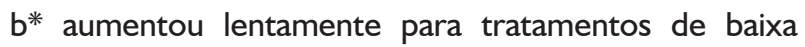

intensidade e diminuiu para tratamentos em que foram aplicadas altas temperaturas. Esses resultados podem ser explicados pelas modificações químicas ocorridas a diferentes temperaturas. Poubel (20II) observou que a coordenada $\mathrm{a}^{*}$ da madeira de Pinus caribaea termorretificada a 180 e $200^{\circ} \mathrm{C}$, durante 2 horas é diretamente proporcional ao teor de extrativos. Mori et al. (2004), encontraram elevada correlação entre a cor 
do lenho de eucalipto e a concentração de polifenóis, extrativos responsáveis pela sua determinação.

Como os extrativos apresentam uma estrutura heterogênea entre as espécies e um número altamente variável de componentes químicos, recomendase estudos específicos para cada espécie, visando a determinar seu efeito na alteração da cor da madeira termorretificada. Nunes (20I2) também observou diferentes comportamentos para as variáveis cromáticas $a^{*}$ e b* quando comparou o efeito do tratamento à 180 e $200^{\circ} \mathrm{C}$ entre as espécies de Corymbia citriodora e Eucalyptus pellita.

De acordo com a classificação de cores proposta por Camargos (1999), a qual baseia-se nas variáveis L*, $a^{*}, b^{*}, C^{*}$ e $h$, a madeira original de Eucalyptus grandis apresenta cor rosa. Para a variável $C^{*}$, o tratamento à $230^{\circ} \mathrm{C}$ por 4 horas apresentou um valor médio de 13,78 para os dois planos analisados (radial e tangencial), correspondendo à cor marrom-escura enquanto que os demais tratamentos termorretificadores apresentaram valores de $C^{*}$ entre 20 e 25 , correspondendo à cor marrom-clara (Tabela I) (Camargos, 1999).

$O$ valor $\mathrm{h}$ corresponde ao ângulo de tinta, com relação as coordenadas $a^{*}$ e b*. Na seção radial, os maiores valores $\mathrm{h}$ foram encontrados para $\mathrm{o}$ tratamento a $200^{\circ} \mathrm{C}$ por 4 horas e o menor para 0 tratamento a $180^{\circ} \mathrm{C}$ por 2 horas (Tabela I). Na seção tangencial, os maiores valores $\mathrm{h}$ foram encontrados para 0 tratamento a 200 e $230^{\circ} \mathrm{C}$ por 4 horas e o menores para os tratamentos a $180^{\circ} \mathrm{C}$ por 2 e 4 horas (Tabela I). $O$ valor $h$ é pouco utilizado para a classificação das cores da madeira.

$\mathrm{Na}$ Figura 2, apresenta-se a variação total da cor $(\Delta \mathrm{E})$ da madeira de Eucalyptus grandis, nas faces radial e tangencial, em função da temperatura e do tempo de termorretificação. Observa-se que a variação total da cor da madeira geralmente aumenta com a temperatura e o tempo, para os tratamentos entre $200 \mathrm{e}$ $230^{\circ} \mathrm{C}$. Geralmente, quando os valores de temperatura se aproximam de $200^{\circ} \mathrm{C}$ a mudança é mais intensa, sendo que a maior parte do escurecimento ocorre nas primeiras quatro horas de exposição (BEKHTA; NIEMZ, 2003). Gouveia (2008), por meio da técnica de reflectância difusa no infravermelho, observou o comportamento de componentes químicos de três espécies de folhosas tratadas termicamente, sob duas condições de temperatura $\left(150\right.$ e $200^{\circ} \mathrm{C}$ ) e tempo (I e 2 horas) em pontos específicos do espectro. $O$ autor verificou que as madeiras submetidas ao tratamento mais agressivo tiveram um aumento de $50 \%$ em suas

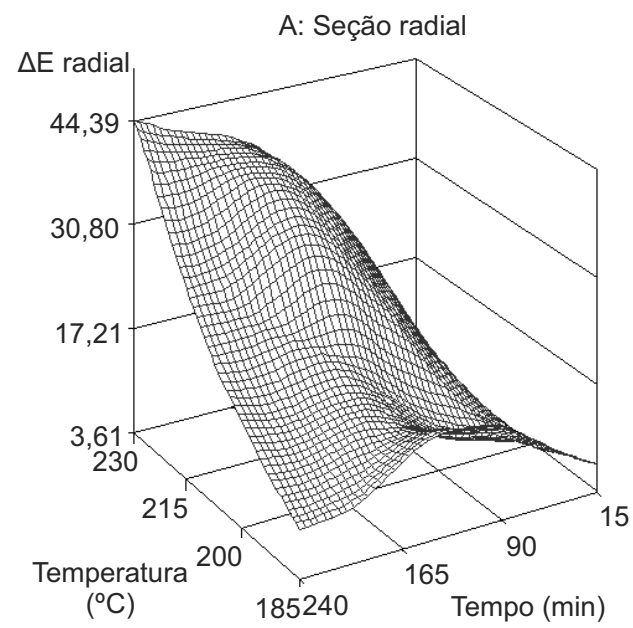

B: Seção tangencial

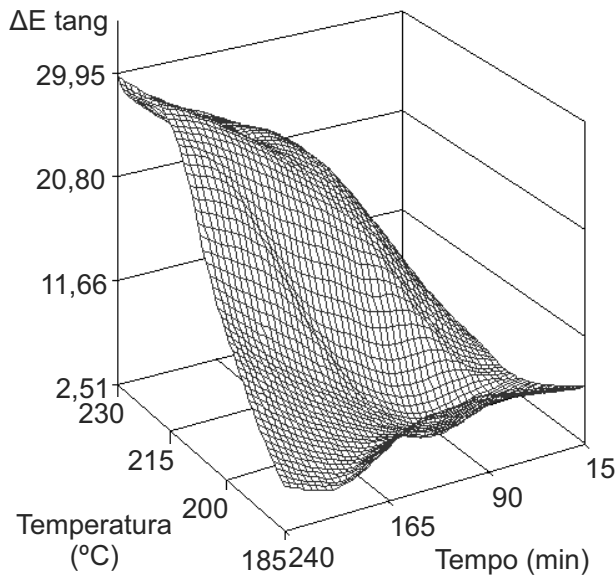

FIGURA 2 Variação total da cor $(\Delta \mathrm{E})$ da madeira de Eucalyptus grandis em função da temperatura e do tempo de termorretificação, nas seções radial e tangencial, respectivamente.

FIGURE 2 Total color variation $(\Delta \mathrm{E})$ of the Eucalyptus grandis wood as a function of temperature and time of heat treatment, on radial and tangential sections, respectively.

ligações $\mathrm{C}=\mathrm{C}$ presentes nos anéis aromáticos os quais representam $\circ$ padrão de intensidade de lignina e extrativos, porém o tratamento provocou uma maior degradação de compostos como carbonilas ligadas ao anel benzênico. Todos os tratamentos apresentaram a formação de cromóforos, a qual foi relativamente maior no tratamento de maior temperatura e tempo.

Entretanto, para $\circ$ tratamento à $180^{\circ} \mathrm{C}$, a variação total da cor é máxima quando a madeira é tratada durante 2 horas, sendo esse resultado influenciado, principalmente, pela variável $L^{*}$. A madeira quando exposta a altas temperaturas durante pouco tempo sua estrutura celular permanece inalterada, porém sofre escurecimento, em razão da pirólise (WINANDY e 
ROWEL, 2005). Segundo o Thermowood ${ }^{\circledR}$ Handbook (2003), o teor de extrativos da madeira aumenta quando o tratamento é realizado a temperaturas de até $180^{\circ} \mathrm{Ce}$ diminui a temperaturas próximas à $230^{\circ} \mathrm{C}$. Isso ocorre porque à baixas temperaturas há uma degradação dos compostos da madeira, principalmente hemiceluloses, enquanto que à altas temperaturas ocorre a volatilização dos extrativos.

Comparando os fatores avaliados (temperatura e tempo), observa-se que a variável temperatura tem um efeito mais pronunciado na alteração da cor. Segundo Garcia et al. (20I2), a temperatura também foi o fator que mais afetou a perda de massa e o teor de umidade de equilíbrio da madeira de Eucalyptus grandis tratada nas mesmas condições desse estudo. Nunes (20I2) encontrou uma relação direta entre a perda de massa e a alteração de cor total $(\Delta \mathrm{E})$ das madeiras de Corymbia citriodora e Eucalyptus pellita após a termorretificação. Nesse caso, a madeira termorretificada de Eucalyptus pellita teve maior perda de massa, provavelmente causada pela degradação das hemiceluloses e volatilização dos extrativos, e também a maior variação de cor. Isso indica que, provavelmente, a alteração total da cor da madeira de Eucalyptus grandis é dependente das alterações químicas ocasionadas pela ação do calor. Segundo Chen et. al (20l2) essas alterações estão associadas ao fenômeno da pirólise, caracterizada pela degradação da madeira na ausência de agentes oxidantes e durante esse processo há formação de produtos oxidativos (ex.: quinonas), produtos da degradação das hemiceluloses e da lignina, os quais migram às superfícies da madeira. Outras alterações ocorrem, tais como, remoção ou migração dos extrativos e compostos nutritivos tais como açúcares de baixo peso molecular e aminoácidos em direção às superfícies da madeira; e aumento no teor de lignina, os quais podem trazer alterações importantes na cor original da madeira (SUNDQVIST; MORÉN, 2002; BEKHTA; NIEMZ, 2003; SEHLSTEDTPERSSON, 2005; ESTEVES et al., 2008).

\section{CONCLUSÕES}

A termorretificação causou um escurecimento (menor valor de $L^{*}$ ) na madeira de Eucalyptus grandis para todas as condições de temperatura e tempo analisadas. Os menores valores de L* foram observados para os tratamentos mais severos, ou seja, aqueles efetuados a altas temperaturas $\left(215\right.$ e $\left.230^{\circ} \mathrm{C}\right)$ e com maior duração (2 e 4 horas).
O efeito da temperatura e do tempo de tratamento nas variáveis cromáticas $a^{*}$ e $b^{*}$ não apresentaram uma tendência definida, ou seja, os valores de $a^{*}$ e b* podem aumentar ou diminuir dependendo das condições de temperatura e tempo.

As madeiras tratadas na condição mais severa de temperatura e tempo $\left(230^{\circ} \mathrm{C}\right.$ por 4 horas $)$ apresentaram os menores valores de L*, a*, b*, caracterizando uma cor marrom-escura, enquanto que nos demais tratamentos, as madeiras foram caracterizadas com uma cor marrom-clara.

A alteração da cor original da madeira após a termorretificação possibilitou a criação de novos padrões de cor, o que pode agregar maior valor a madeira estudada.

Estudos sobre as modificações químicas da madeira termorretificada são necessários, a fim de explicar os fenômenos envolvidos no processo de formação da cor da madeira sob diferentes condições de temperatura e tempo de termorretificação.

\section{AGRADECIMENTOS}

Os autores agradecem ao Professor Dr. Joaquim C. Gonçalez do Departamento de Engenharia Florestal da Universidade de Brasília pelo apoio técnico.

\section{REFERÊNCIAS}

AHAJJI, A.; DIOUF, P. N.; ALOUI, F.; ELBAKALI, I.; PERRIN, D.; MERLIN, A.; GEORGE, B. Influence of heat treatment on antioxidant properties and colour stability of beech and spruce wood and their extractives. Wood Science and Technology, New York, v. 43, p. 69-83, 2009.

AKYILDIZ, M. H.; ATES, S.; ÖZDEMIR, H. Technological and chemical properties of heat-treated Anatolian black pine wood. African Journal of Biotechnology, Nairobi, v. 8, n. II, p. 2565-2572, 2009.

AYADI, N.; LEJEUNE, F.; CHARRIER, F.; CHARRIER, B.; MERLIN, A. Color stability of heat-treated wood during artificial weathering. Holz als Roh-und Werkstoff, Berlin, v. 6I, p. 22 I-226, 2003.

BEKHTA, P.; NIEMS, P. Effect of high temperature on the change in colour, dimensional stability and mechanical properties of spruce wood. Holzforschung, Berlin, v. 57 , p. 539-546, 2003.

BOURGOIS, P. J.; JANIN, G.; GUYONNET, R. The color measurement: a fast method to study and to optimize the chemical transformations undergone in the thermally treated wood. Holzforschung, Berlin, v. 45, p. 377-382, I99I. 
CAMARGOS, J. A. A. Colorimetria quantitativa aplicada na elaboração de um catálogo de cores de madeiras tropicais. 1999. 75 f. Dissertação (Mestrado em Ciências Florestais) - Universidade de Brasília, Brasília, 1999.

CHEN, Y.; FAN, Y.; GAO, J.; LI, H. Coloring characteristics of in situ lignin during heat treatment. Wood Science Technology, New York, v. 46, n. I, p. 33-40, 2012.

CHEN, Y.; FAN, Y.; GAO, J.; LI, H. The effect of heat treatment on the chemical and color change of black locus (Robinia pseudoacacia) wood flour. BioResources, Raleigh, v. 7, n. I, p. II57-II70, 2012.

DUBEY, M. K.; PANG, S.; WALKER, J. Effect of oil heating age on colour and dimensional stability of heat treated Pinus radiata. European Journal of Wood and Wood Products, München, v. 69, p. 255-262, 201 I.

ESTEVES, B.; VELEZ MARQUES, A.; DOMINGOS, I.; PEREIRA, H. Heat induced colour changes of pine (Pinus pinaster) and eucalyptus (Eucalyptus globulus) wood. Wood Science and Technology, New York, v. 42, n. 5, p. 369384, 2008.

FINNISH THERMOWOOD ASSOCIATION. ThermoWood handbook. Helsinki, 2003. 66 p.

GARCIA, R. A.; CARVALHO, A. M.; LATORRACA, J. V. F; MATOS, J. L. M.; SANTOS, W. A.; SILVA, R. F. M. Nondestructive evaluation of heat-treated Eucalyptus grandis Hill ex. Maiden wood using stress wave method. Wood Science and Technology, New York, v. 46, p. 4I$52,2012$.

GONÇALEZ, J. C.; BREDA, L. de C. S.; BARROS, J. F. M.; MACEDO, D. G.; JANIN, G.; COSTA, A. F. da; VALE, A. T. Características tecnológicas das madeiras de Eucalyptus grandis W. Hill ex Maiden e Eucalyptus cloeziana F. Muell visando ao seu aproveitamento na indústria moveleira. Ciência Florestal, Santa Maria, v. 16, n. 3, p. 329-34I, 2006.

GONZÁLEZ-PENA, M. M.; HALE, M. D. C. Colour in thermally modified wood of beech, Norway spruce and Scots pine part I: colour evolution and colour changes. Holzforschung, Berlin, v. 63, p. 385-393, 2009.

GOUVEIA, F. N. Aplicação de tratamentos térmicos para estabilização colorimétricas de madeiras tropicais. 2008. 130 p. Tese (Doutorado em Ciência Florestal) Universidade de Brasília, Brasília, 2008.

ISHIGURI, F.; MARUYAMA, S.; TAKAHASHI, K.; ABE, Z.; YOKOTA, S.; ANDOH, M.; YOSHIZAWA, N. Extractives relating to heartwood color changes in sugi (Crytomeria japonica) by a combination of smoke-heating and UV radiation exposure. Journal of Wood Science, Kyoto, v. 49, p. 135-139, 2003.
KAMDEM, D. P.; PIZZI, A.; JERMANNAUD, A. Durability of heat-treated wood. Holz als Roh-und Werkstoff, Berlin, v. 60 , p. I-6, 2002

KAMKE, F. A. Densified radiata pine for structural composites. Maderas. Ciencia y Tecnología, Concepción, v. 8, n. 2, p. 83-92, 2006.

KONICA MINOLTA SENSING. Comunicação precisa da cor: controle de qualidade da percepção à instrumentação. São Paulo, 1998. 59 p.

MILITZ, R. Heat treatment technologies in Europe: scientific background and technological state-of-art. In: CONFERENCE ON ENHANCING THE DURABILITY OF LUMBER AND ENGINEERED WOOD PRODUCTS, I., 2002, Kissimmee. Proceedings... Madison: Forest Products Society, 2002. I CD-ROM.

MITSUI, K. Changes in color of spruce by repetitive treatment of light-irradiation and heat treatment. Holz als Roh-und Werkstoff, Berlin, v. 64, p. 243-244, 2006.

MITSUI, K. Changes in the properties of light-irradiated wood with heat treatment: part 2, effect of light-irradiation time and wavelength. Holz als Roh-und Werkstoff, Berlin, v. 62, p. 23-30, 2004.

MITSUI, K.; TAKADA, H.; SUGIYAMA, M.; HASEGAWA, R. Changes in the properties of light-irradiated wood with heat treatment: part I, effect of treatment conditions on the change in color. Holzforschung, Berlin, v. 55, p. 60I-605, $200 \mathrm{I}$

MORI, C. L. S. O.; MORI, F. A.; LIMA, J. T.; TRUGILHO, P. F.; OLIVEIRA, A. C. Influência das características tecnológicas na cor da madeira de eucaliptos. Ciência Florestal, Santa Maria, v. 14, n. 2, p. 123-132, 2004.

MOURA, L. F. de; BRITO, J. O. Effect of thermal rectification on colorimetric properties of Eucalyptus grandis and Pinus caribaea var. hondurensis woods. Scientia Forestalis, Piracicaba, v. 39, n. 89, p. 69-76, 2011.

NUNES, C. S. Propriedades tecnológicas e qualidade de adesão de madeiras de Corymbia citriodora e Eucalyptus pellita termorretificadas. 2012. 56 p. Dissertação (Mestrado em Ciências Ambientais e Florestais) - Universidade Federal Rural do Rio de Janeiro, Seropédica, 2012.

NUOPPONEN, M.; VUORINEN, T.; JÄMSÄ, S.; VIITANIEMI, $P$. The effects of a heat treatment on the behaviour of extractives in softwood studied by FTIR spectroscopic methods. Wood Science and Technology, New York, v. 37, p. 109-I I5, 2005.

OYARCE, C. D. B. Modification of wood color via electroheating technology. 2006. 56 f. Dissertation (Master of Science) - University of Idaho, Idaho, 2006. 
PATZELT, M.; EMSENHUBER, G.; STINGL, R. Colour measurement as means of quality control of thermally treated wood. In: EUROPEAN CONFERENCE ONWOOD MODIFICATION, I., 2003, Ghent. Proceedings... Ghent: ECWM, 2003. p. 213-218.

POUBEL, D. S. Efeito da termorretificação nas propriedades tecnológicas da madeira normal e de compressão de Pinus caribaea Morelet. 20ll. $40 \mathrm{f}$. Monografia (Curso de Engenharia Florestal) - Universidade Federal Rural do Rio de Janeiro, Seropédica, 201 I.

RIBEIRO, P. G. Utilização de técnicas não destrutivas para caracterização de madeiras de Pinus caribaea var. hondurensis e de Eucalyptus grandis. 2009. Il4 f. Dissertação (Mestrado em Ciências Florestais) Universidade de Brasília, Brasília, 2009.

SCHNABEL, T.; ZIMMER, B.; PETUTSCHNIGG, A. J.; SCHÖNBERGER, S. An approach to classify thermally modified hardwoods by color. Forest Products Journal, Madison, v. 57, n. 9, p. I05-I I0, 2007.

SEHLSTEDT-PERSSON, M. Properties of solid wood: responses to drying and heattreatment. luleå university of technology. 2005. 142 f. Thesis (Doctor in Wood Science and Technology) - Luleå University of Technology, Skellefteå, 2005.
SUNDQVST, B. Colour changes and acid formation in wood during heating. 2004. I54 f. Thesis (Doctor in Wood Material Science) - Luleå University of Technology, Skelleftå, 2004.

SUNDQVIST, B.; MORÉN, T. The influence of wood polymers and extractives on wood colour induced by hydrothermal treatment. Holz als Roh-und Werkstoff, Berlin, v. 60, p. 375-376, 2002.

THEANDER, O.; BJURMAN, J.; BOUTELJE, J. B. Increase in the content of low-molecular carbohydrates at lumber surfaces during drying and correlations with nitrogen content, yellowing and mould growth. Wood Science and Technology, New York, v. 27, n. 5, p. 38I-389, 1993.

TJEERDSMA, B. F.; BOONSTRA, M.; PIZZI, A.; TEKELY, P.; MILLITZ, $H$. Characterization of thermally modified wood: Molecular reasons for wood performance improvement. Holz als Roh-und Werkstoff, Berlin, v. 56, p. I49-I53, 1998.

VARGA, D.; ZEE, M. E. van der. Influence of steaming on selected wood properties of four hardwood species. Holz als Roh-und Werkstoff, Berlin, v. 66, p. II-I8, 2008.

WINANDY, J. E.; ROWELL, R. M. Chemistry of wood strength. In: Handbook of wood chemistry and wood composites. Boca Raton: Rowell, 2005. p. 329-330. 
